\title{
Effect of thermal boundary conditions on conjugate natural convection flow in vertical eccentric annuli
}

\author{
A. Jamal ${ }^{1}$, M. A. I. El-Shaarawi ${ }^{2}$ \& E. M. A. Mokheimer ${ }^{2}$ \\ ${ }^{I}$ Department of Aerospace Engineering, \\ King Fahd University of Petroleum and Minerals, Saudi Arabia \\ ${ }^{2}$ Department of Mechanical Engineering, \\ King Fahd University of Petroleum and Minerals, Saudi Arabia
}

\begin{abstract}
The effect of thermal boundary conditions on conjugate laminar natural convection heat transfer in vertical eccentric annuli is numerically investigated using the finite-difference technique. Numerical results are presented for a Newtonian fluid of Prandtl number 0.7 in an eccentric annulus. The variation of induced flow rate and total heat absorbed in the annulus are studied for two sets of boundary conditions at different values of geometry parameters (dimensionless annulus eccentricity and radius ratio). In both sets of boundary conditions, one wall is heated isothermally. The other wall is kept at the inlet fluid temperature for the first set of boundary conditions and adiabatic for the second set. The effect of interchanging the wall thermal conditions for each set is also considered. Analysis reveals that heating the outer cylinder wall or keeping one of the annulus walls insulated is more useful for inducing flow (thermosiphons).
\end{abstract}

Keywords: natural convection, heat transfer, eccentric annuli, thermal boundary conditions, finite difference method, Newtonian fluid.

\section{Introduction}

The study of steady laminar induced flow in vertical eccentric annuli with conjugate heat transfer is of great importance because of its many engineering applications in electrical, nuclear, solar and thermal storage fields. In the 
electrical field, in vertical electric motors and generators, the heat generated by irreversible electrical and mechanical processes is transferred through the air gap between the rotor and the stator by natural convection. The transfer of heat by free convection is always a factor in the cooling of such machines and may be the sole means of cooling small types of these devices.

Considerable work has been done to study the problem of flow and conjugate heat transfer in various geometries and annuli, both concentric and eccentric. Anand and Tree [1] studied the effect of axial conduction in a tube wall on the steady-state laminar convective heat transfer. Using finite-difference technique, El-Shaarawi and Negm [2] solved the laminar conjugate natural convection problem in vertical open-ended concentric annuli. Similarly, in spite of the many studies reported in the literature for the conventional case of convection in eccentric annuli [3-5], the only work available for the conjugate case is that of El-Shaarawi and Haider [6] for forced convection. They presented results for a fluid of Prandtl number $P r=0.7$ flowing in an annulus of radius ratio $N R_{2}=0.5$ with eccentricity $E=0.1,0.3,0.5$, and 0.7 . Moreover, in a recent paper ElShaarawi et al [7] presented some results for the conjugate heat transfer in the free convection regime.

The literature survey summarized above revealed that conjugate natural convection heat transfer in vertical eccentric annuli is still in its infancy stage. The present paper presents a boundary layer model for the problem of developing steady laminar conjugate natural convection heat transfer in vertical eccentric annuli. A numerical algorithm, employing finite difference technique, is developed to solve the obtained model. Numerical results are presented to show the effects of thermal boundary conditions on the conjugate natural convection flow and heat transfer in vertical eccentric annuli at different values of geometry parameters (dimensionless annulus eccentricity $(E)$ and radius ratio $\left(N R_{2}\right)$ ). Two sets of boundary conditions are considered in the present analysis. In both sets of boundary conditions, one wall is heated isothermally. The other wall is kept at inlet fluid temperature for the first set of boundary conditions and adiabatic for the second set. Furthermore, the effect of switching the walls thermal conditions for each set is also analyzed.

\section{Problem formulation}

The vertical eccentric annulus of finite height and thickness, as shown in fig. 1, is open at both ends and is immersed in a stagnant Newtonian fluid maintained at constant temperature $\left(T_{o}\right)$. Free convection flow is induced inside this annular channel as a result of heating one of the channel walls isothermally while keeping the other wall at inlet fluid temperature (case 1) or adiabatic (case 2). In both cases, 'I' refers to the case when the heat transfer wall is the inner cylinder wall and ' $\mathrm{O}$ ' vice versa. Thus, case (1.I) corresponds to the thermal boundary condition having the inner cylinder wall isothermally heated while the outer wall kept at inlet fluid temperature. It is evident from fig. 1 that the eccentric annular geometry is symmetric about line $\mathrm{AB}$, therefore, only the half symmetric section is taken for the analysis. 


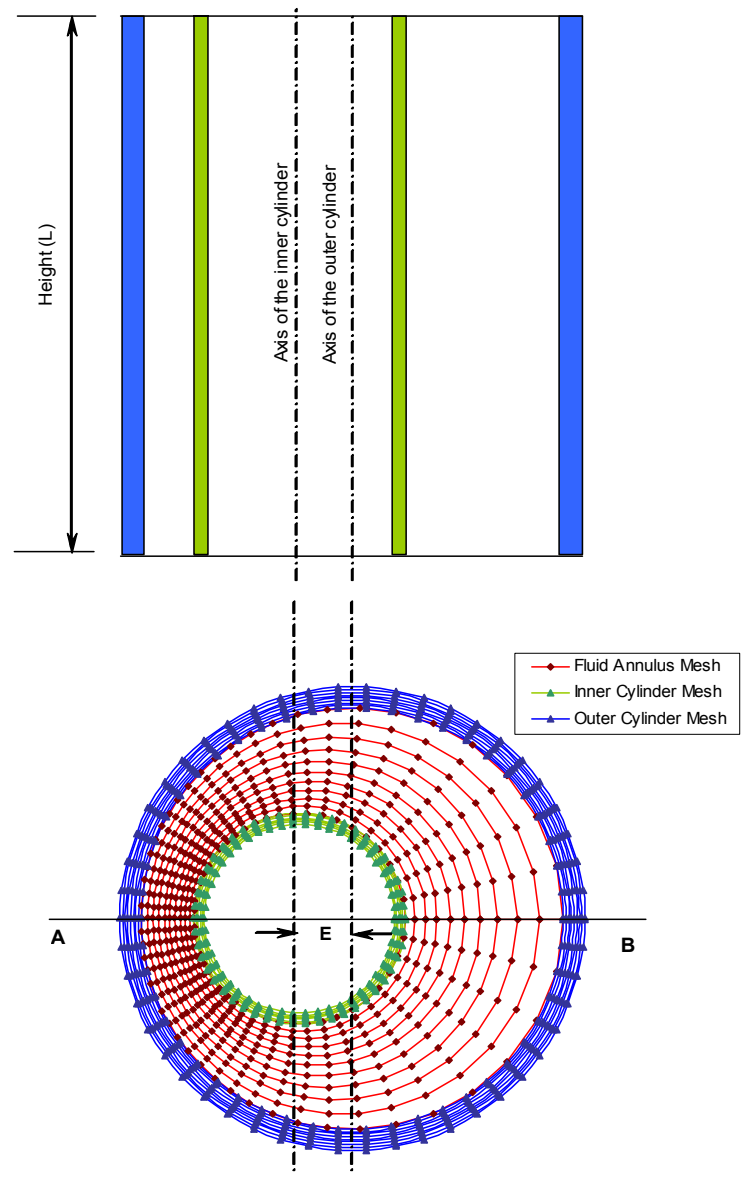

Figure 1: Elevation and numerical mesh of the eccentric annulus.

The flow is steady, laminar, enters the eccentric annulus with a uniform velocity distribution $\left(U_{o}\right)$. Body forces in other than the vertical direction, viscous dissipation $(\Phi)$, internal heat generation $\left(Q^{\prime \prime \prime}\right)$ and radiation heat transfer are absent. The governing equations describing flow and heat transfer through the eccentric annulus are the conservation equations of mass, momentum and energy given in a general orthogonal curvilinear coordinate system by Hughes and Gaylord [8].

The bipolar coordinate system is more suitable to express the partial differential equations describing the flow and heat transfer through the vertical eccentric annulus, shown in fig. 1. On the other hand, the cylinder walls have uniform thickness. Hence, the cylindrical coordinate system is more appropriate for the solid walls. Some parabolic-flow assumptions by El-Shaarawi and 
Mokheimer [9] will be used to simplify the governing equations. The assumptions include: the pressure is a function of the axial coordinate only $\left(\frac{\partial p}{\partial \eta}=\frac{\partial p}{\partial \xi}=0\right)$, the axial diffusions of momentum and energy are neglected $\left(\frac{\partial^{2}}{\partial z^{2}}=0\right)$, and the $\eta$-velocity component $(v)$ is much smaller than the $\xi$ and $z$ velocity components ( $w$ and $u$ ). Introducing the dimensionless parameters given in the nomenclature, carrying out an order of magnitude analysis and taking into consideration that the latter assumption results in dropping the $\eta$-momentum equation, the governing equations are:

Continuity equation

$$
\frac{\partial(H W)}{\partial \xi}+\frac{\partial(H V)}{\partial \eta}+4\left(1-N R_{2}\right)^{2} \frac{\partial\left(U H^{2}\right)}{\partial Z}=0
$$

Momentum equation in Z-direction

$$
\begin{aligned}
\frac{W}{H} \frac{\partial U}{\partial \xi}+\frac{V}{H} \frac{\partial U}{\partial \eta}+4\left(1-N R_{2}\right)^{2} U \frac{\partial U}{\partial Z} & =\frac{\theta}{4\left(1-N R_{2}\right)^{2}}-\frac{1}{4\left(1-N R_{2}\right)^{2}} \frac{\partial P}{\partial Z}+ \\
& \frac{1}{H^{2}}\left(\frac{\partial^{2} U}{\partial \eta^{2}}+\frac{\partial^{2} U}{\partial \xi^{2}}\right)
\end{aligned}
$$

Momentum equation in $\xi$-direction

$$
\begin{aligned}
& \frac{W}{H} \frac{\partial W}{\partial \xi}+\frac{V}{H^{2}} \frac{\partial(H W)}{\partial \eta}+4\left(1-N R_{2}\right)^{2} U \frac{\partial W}{\partial Z}-\frac{V^{2}}{H^{2}} \frac{\partial H}{\partial \xi}=-\frac{G r^{2}}{H} \frac{\partial P}{\partial \xi}+ \\
& \frac{1}{H^{3}}\left[\frac{\partial^{2}(H W)}{\partial \eta^{2}}+\frac{\partial^{2}(H W)}{\partial \xi^{2}}\right]+\frac{1}{H G r^{2}} \frac{\partial^{2}(H W)}{\partial Z^{2}}- \\
& \frac{2}{H^{4}}\left[\frac{\partial(H W)}{\partial \eta}-\frac{\partial(H V)}{\partial \xi}\right] \frac{\partial H}{\partial \eta}+\frac{8\left(1-N R_{2}\right)^{2}}{H^{2}} \frac{\partial H}{\partial \xi} \frac{\partial U}{\partial Z}
\end{aligned}
$$

Energy equation for fluid

$$
\frac{W}{H} \frac{\partial \theta_{f}}{\partial \xi}+\frac{V}{H} \frac{\partial \theta_{f}}{\partial \eta}+4\left(1-N R_{2}\right)^{2} U \frac{\partial \theta_{f}}{\partial Z}=\frac{1}{\operatorname{Pr} H^{2}}\left(\frac{\partial^{2} \theta_{f}}{\partial \eta^{2}}+\frac{\partial^{2} \theta_{f}}{\partial \xi^{2}}\right)
$$

Energy equation for solid

$$
\frac{\partial^{2} \theta_{s}}{\partial R^{2}}+\frac{1}{R} \frac{\partial \theta_{s}}{\partial R}+\frac{1}{R^{2}} \frac{\partial^{2} \theta_{s}}{\partial \phi^{2}}=0
$$

The thermal boundary conditions considered in this investigation are:

For outer cylinder, $\theta_{s}=\theta_{s o} \& R$ vary from $N R_{3}=1$ to $N R_{4}$

For inner cylinder, $\theta_{s}=\theta_{s i} \& R$ vary from $N R_{1}$ to $N R_{2}$ 
Integral form of the continuity equation

$$
\bar{U}=\frac{8\left(1-N R_{2}\right)}{\pi\left(1+N R_{2}\right)} \int_{0}^{\pi} \int_{\eta_{O}}^{\eta_{I}} U H^{2} d \eta d \xi
$$

Having the governing equations for the fluid in bipolar coordinates and the energy equations for the solid walls in cylindrical coordinates generates unmatched grid points on both the interfaces. Therefore, these points are linked to determine the temperatures at the two interfaces by applying the principles of continuity of temperature and continuity of heat flux at these interfaces. Equations (1-6), subject to boundary conditions, have been numerically solved as indicated in [7].

\section{Results and discussion}

Numerical results have been obtained for cases (1.I), (1.O), (2.I), and (2.O) for dimensionless eccentricities, $E=0.1,0.3,0.5$, and 0.7 and radius ratios, $N R_{2}=0.1$, $0.3,0.5$, and 0.7 at given solid-fluid conductivity ratio $(K R=10)$, cylinder wall thicknesses $\left(\delta_{i}\right.$ and $\delta_{o}=0.1$ and 0.2$)$, and Prandtl number $(P r=0.7)$.

In the present analysis, a grid of 25 segments in each $\eta$ and $\xi$ directions in the fluid annulus whereas 20 and 10 segments in the r-direction in the outer and inner cylinder walls, respectively, and 25 segments in $\phi$-direction in each of the cylinder walls are used (Jamal [10]). To check the adequacy of the present computer code, special runs were carried out simulating the two different limiting cases of conventional and conjugate convection for the given eccentric annuli. The results of these special computer code experimentations are as follows.

First, a special computer run simulating the conventional natural convection case was done. The obtained results for the channel height required to suck specific flow rates for case (1.I) are compared in fig. 2 with the corresponding results of El-Shaarawi and Mokheimer [9]. The maximum error never exceeded $2.87 \%$.

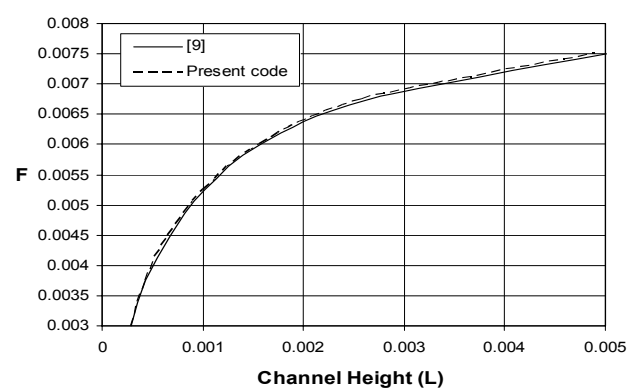

Figure 2: Comparison of $F$ versus $L$ as reported by El-Shaarawi and Mokheimer [9] and the corresponding results by a special computer run simulating conventional free convection. 
Secondly, the present computer code was validated for the conjugate forced convection case in eccentric annuli by comparing the results obtained from a pertaining special run with the corresponding developing and fully developed temperature profiles across the widest gap $(\Psi=0)$ of El-Shaarawi and Haider [6]; excellent agreement was observed as the maximum deviation between the obtained results and those of [6] never exceeded $0.23 \%$.

Owing to the space limitations, only a representative sample of the results will be presented. Figure 3 presents the variation of induced flow rate $(F)$ with the channel height $(L)$ for cases (1.I) and (1.O) at different values of dimensionless eccentricity $(E)$. It can be seen from the comparison that at aiven $L$ and $E, F$ is greater for case (1.O) than that for case (1.I). This can be attributed to the larger heated surface area for case (1.O) than for case (1.I) that enables more amount of heat to flow into the fluid annulus and causes higher mean fluid temperature and buoyancy force in the annulus, hence resulting in higher values of $F$. In the figure, the dominant effect of heating the outer wall is observed.

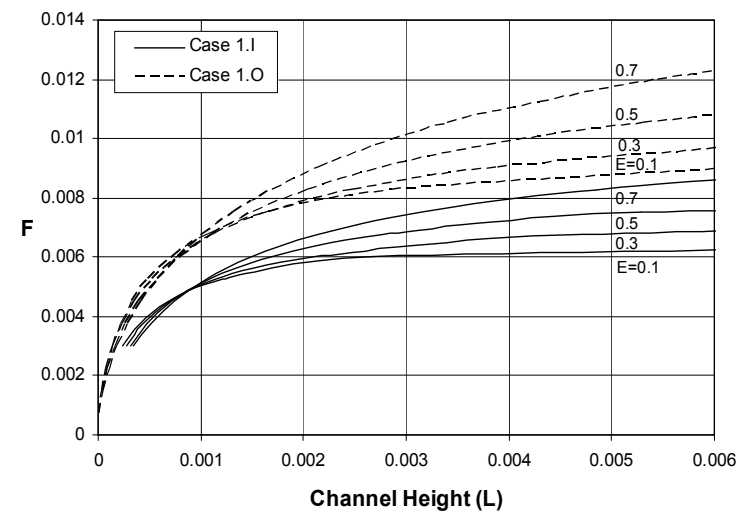

Figure 3: Comparison of flow rate with channel height for different values of eccentricity among cases (1.I) and (1.O).

Relative comparison of $F$ versus $L$ is presented for cases (1.O) and (2.O) at different values of radius ratio $\left(N R_{2}\right)$ in fig. 4 . Due to the presence of one insulated wall in case (2.O), heat finds no way to flow through other than the fluid. This allows more amount of heat to be absorbed by the fluid thus raising the mean fluid temperature and leading to a higher value of $F$ than for case (1.O). Similar behavior has been observed in other comparisons between the two boundary conditions $(1 \& 2)$ at different values of $E$ and $N R_{2}$.

Figure 5 shows the variation of total heat absorbed $(\bar{Q})$ versus $L$ for cases (1.I) and (2.I) at different values of $E$. Similar plot is obtained for cases (1.I) and (1.O) at different values of $N R_{2}$ as shown in fig. 6. Since $\bar{Q}$ is directly related to $F$ through the relation, $\bar{Q}=F \theta_{m, e x}$, therefore, behavior similar to figs. 3 and 4 
has been observed, i.e., case $(\mathrm{O})$ of both the boundary conditions ( $1 \& 2$ ) shows higher values of $\bar{Q}$ than case (I) and boundary condition (2) (I \& O) shows higher values of $\bar{Q}$ than boundary condition (1), at any given $L, E$, and $N R_{2}$.

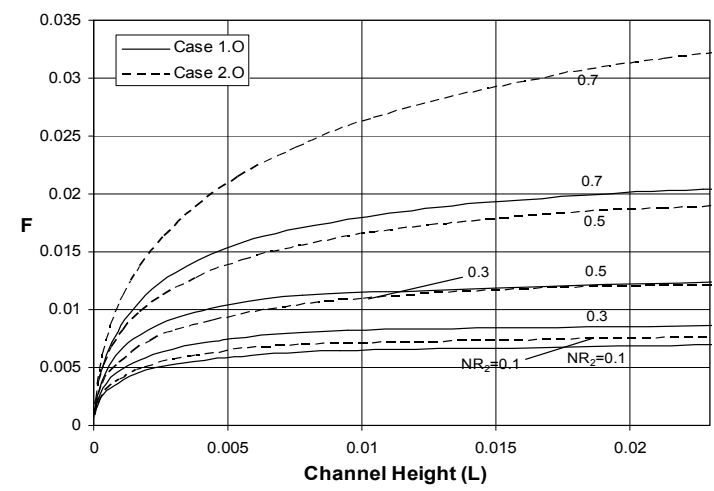

Figure 4: Comparison of flow rate with channel height for different values of radius ratio among cases (1.O) and (2.O).

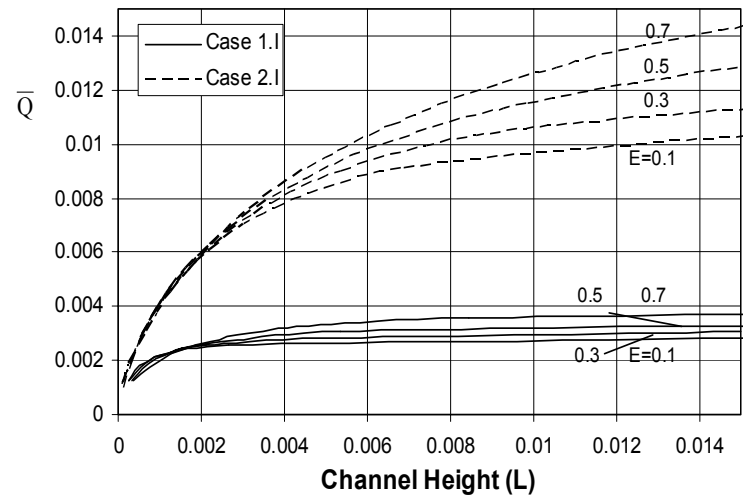

Figure 5: Comparison of total heat absorbed for different values of eccentricity among cases (1.I) and (3.I).

\section{Conclusions}

Combined conduction-laminar free convection heat transfer in vertical eccentric annuli has been numerically investigated. A finite-difference algorithm has been developed to solve the bipolar model equations. Numerical results are presented for a fluid of Prandtl number, $P r=0.7$ in an eccentric annulus. The effect of boundary conditions on the variations of the induced flow rate $(F)$ and the heat absorbed $(\bar{Q})$ in the eccentric annulus has been investigated at different values of eccentricity $(E)$ and radius ratio $\left(N R_{2}\right)$. 

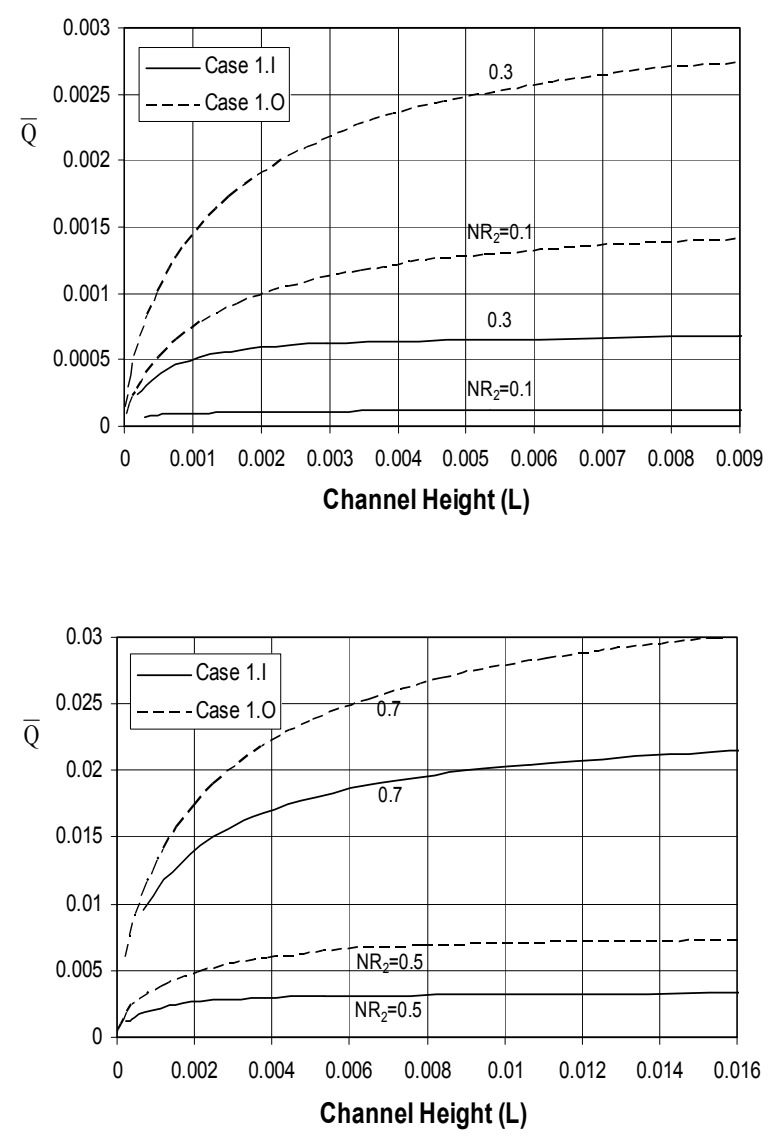

Figure 6: Comparison of total heat absorbed for different values of eccentricity among cases (1.I) and (2.I).

The results of the present work have revealed that cases such as (1.O) and (2.O) have higher values of $F$ and $\bar{Q}$ than cases (1.I) and (2.I), respectively. Similarly, the values of $F$ and $\bar{Q}$ are higher for cases (2.I) and (2.O) than for cases (1.I) and (1.O), respectively. Hence it can be inferred from the results that the heat transfer is more pronounced for case $(\mathrm{O})$ and boundary condition (2) and heating the outer cylinder wall or keeping one of the annulus walls insulated is more useful for inducing flow (thermo-siphons).

\section{Nomenclature}

$D_{h} \quad$ Hydraulic or equivalent $\quad G r^{*}$ Modified Grashof number diameter of annulus, $\mathrm{m}$ 
$H$ Dimensionless coordinate transformation scale factor

$M \quad$ No. of intervals in each of the $\xi \& \phi$-directions

$N \quad$ Number of intervals in the $\eta$ direction

$N R_{1} \quad$ Ratio between inner radius of inner cylinder and inner radius of outer cylinder, $\frac{r_{i i}}{r_{i o}}$

$\mathrm{NR}_{2}$ Ratio between outer radius of inner cylinder and inner radius of outer cylinder (Fluid annulus radius ratio), $\frac{r_{o i}}{r_{i o}}$

$N R_{3}$ Dimensionless inner radius of outer cylinder, $\frac{r_{i o}}{r_{i o}}=1$

$N_{4}$ Ratio between outer radius of outer cylinder and inner radius of outer cylinder, $\frac{r_{o o}}{r_{i o}}$

$P$ Dimensionless Pressure defect of fluid inside the channel at any cross section

$r_{i i}$ Inner radius of inner cylinder, $\mathrm{m}$

$r_{o i} \quad$ Outer radius of inner cylinder, $\mathrm{m}$

$r_{i o}$ Inner radius of outer cylinder, $\mathrm{m}$

$r_{o o}$ Outer radius of outer cylinder, $\mathrm{m}$

$R$ Dimensionless radial coordinate, $\frac{r}{r_{i o}}$

$T_{w} \quad$ Isothermal temperature of heated wall, $\mathrm{K}$
$U \quad$ Dimensionless axial velocity at any point, $\frac{u r_{i o}{ }^{2}}{l \gamma G r *}$

$V$ Velocity vector or dimensionless $\quad \eta$-velocity component, $\frac{v D_{h}}{\gamma}$

$W \quad$ Dimensionless $\quad \xi$-velocity component, $\frac{w D_{h}}{\gamma}$

Z Dimensionless axial coordinate in both the Cartesian and bipolar coordinate systems, $\frac{z}{l G r^{*}}$

Greek Letters

$\eta \quad$ First transverse bi-polar coordinate

$\theta$ Dimensionless temperature, $\frac{\left(T-T_{o}\right)}{\left(T_{w}-T_{o}\right)}$ for isothermal walls case

$\theta_{f} \quad$ Value of $\theta$ in the fluid annulus

$\theta_{m, e x}$ Mean fluid temperature at channel exit

$\theta_{s i} \quad$ Value of $\theta$ in the inner solid wall

$\theta_{\text {so }} \quad$ Value of $\theta$ in the outer solid wall.

$\delta_{i} \quad$ Dimensionless thickness of inner cylinder wall, $N R_{2}-N R_{1}$

$\delta_{o} \quad$ Dimensionless thickness of outer cylinder wall, $\mathrm{NR}_{4}-\mathrm{NR}_{3}$

$\phi \quad$ Angle along the cylinder walls

$\xi \quad$ Second transverse bi-polar point.

$\Psi \quad$ Normalized value of $\xi, \frac{\xi}{\pi}$ 


\section{Acknowledgement}

The support of King Fahd University of Petroleum and Minerals to carry out this investigation is gratefully acknowledged.

\section{References}

[1] Anand, N. K. \& Tree, D. R., Some studies of the effects of axial conduction in a tube wall on the steady-state laminar convective heat transfer. Journal of Heat Transfer, 109, pp. 1025-1028, 1987.

[2] El-Shaarawi, M.A.I. and Negm, A. A. A., Transient combined natural convection-conduction in open-ended vertical concentric annuli. Heat and Mass Transfer, 35, pp. 133-141, 1999.

[3] Feldman, E.E., Hornbeck, R.W. and Osterle, J. F., A numerical solution of temperature for laminar developing flow in eccentric annular ducts. International Journal of Heat and Mass Transfer, 25 (2), pp. 243-253, 1982.

[4] El-Shaarawi, M.A.I., Mokheimer, E. M.A., Free convection in vertical eccentric annuli with a uniformly heated boundary. International Journal of Numerical Methods for Heat and Fluid Flow, 8 (5), pp. 488-503, 1998.

[5] Kumar, D.S., Laxminarayana, B. and Balaji, C., Laminar natural convection in cylindrical annuli filled with a low Prandtl number fluid. Heat and Technology, 20 (2), pp. 67-74, 2002.

[6] El-Shaarawi, M.A.I. and Haider, S. A., Critical conductivity ratio for conjugate heat transfer in eccentric annuli. International Journal of Numerical Methods for Heat and Fluid Flow, 11 (2), pp. 255-277, 2001.

[7] El-Shaarawi, M.A.I., Mokheimer, E. M. A. and Jamal, A., Conjugate effects on steady laminar natural convection heat transfer in vertical eccentric annuli. International Journal for Computational Methods in Engineering Science and Mechanics, 6, pp. 235-250, 2005.

[8] Hughes, W.F. and Gaylord, E.W., Basic Equations of Engineering Science, Schaum Outline Series, pp. 150-151, 1964.

[9] El-Shaarawi, M.A.I., Mokheimer, E. M.A., Developing free convection in open ended vertical eccentric annuli with isothermal boundaries. Journal of Heat Transfer, Transaction ASME, 121 (1), pp. 63-72, 1999.

[10] Jamal, A., Conjugate Free Convection Heat Transfer in Vertical Eccentric Annuli, MS Thesis, Mechanical Engineering Department, King Fahd University of Petroleum and Minerals (KFUPM): Dhahran, Saudi Arabia, pp. 52-57, 2002. 